\title{
Bulk Handling Facility Modeling and Simulation for Safeguards Analysis
}

\author{
Benjamin B. Cipiti $(1)$ and Nathan Shoman $(\mathbb{D}$ \\ Sandia National Laboratories, Albuquerque, NM 87185, USA \\ Correspondence should be addressed to Benjamin B. Cipiti; bbcipit@sandia.gov
}

Received 3 July 2018; Accepted 17 September 2018; Published 4 October 2018

Guest Editor: Tom Burr

Copyright (C) 2018 Benjamin B. Cipiti and Nathan Shoman. This is an open access article distributed under the Creative Commons Attribution License, which permits unrestricted use, distribution, and reproduction in any medium, provided the original work is properly cited.

\begin{abstract}
The Separation and Safeguards Performance Model (SSPM) uses MATLAB/Simulink to provide a tool for safeguards analysis of bulk handling nuclear processing facilities. Models of aqueous and electrochemical reprocessing, enrichment, fuel fabrication, and molten salt reactor facilities have been developed to date. These models are used for designing the overall safeguards system, examining new safeguards approaches, virtually testing new measurement instrumentation, and analyzing diversion scenarios. The key metrics generated by the models include overall measurement uncertainty and detection probability for various material diversion or facility misuse scenarios. Safeguards modeling allows for rapid and cost-effective analysis for Safeguards by Design. The models are currently being used to explore alternative safeguards approaches, including more reliance on process monitoring data to reduce the need for destructive analysis that adds considerable burden to international safeguards. Machine learning techniques are being applied, but these techniques need large amounts of data for training and testing the algorithms. The SSPM can provide that training data. This paper will describe the SSPM and its use for applying both traditional nuclear material accountancy and newer machine learning options.
\end{abstract}

\section{Introduction}

Safeguards modeling for fuel cycle facilities requires a systems level view. The SSPM $[1,2]$ was developed to provide a platform for designing new safeguards systems, evaluating the performance of improved measurement instrumentation, and performing diversion scenario analysis. Materials accountancy measurements are highly dependent on the elemental and isotopic compositions as well as the timing sequence of operations. Therefore, it is important to have facility models that adequately model the unit operations, timing, and isotopic material flows. The SSPM provides this capability.

Traditional safeguards approaches involve setting up a material balance across material balance areas (MBAs). This typically requires a significant amount of sampling and destructive analysis (DA) in a laboratory. Statistical tests are used to examine responsiveness to material loss. Recently, there has been increased interest in reducing the burden of laboratory analysis through more use of unattended or process monitoring data to draw safeguards conclusions. However, the use of more process monitoring data requires different safeguards approaches that require modeling and simulation.

\section{Separation and Safeguards Performance Model (SSPM)}

The SSPM is built in MATLAB/Simulink. A PUREX SSPM version is shown in Figure 1. The gray blocks represent the processing vessels throughout the plant and contain significant detail about inventories, timing of operations, filling/emptying sequences, etc. The fuel feed is linked to a database derived from ORIGEN/SCALE [3] calculations to allow the user to choose from a wide variety of spent fuel types. The signals connecting the blocks contain the elemental and isotopic mass flow information of all nuclear material and bulk flows. The model has also been linked to GADRAS [4] (Gamma Detector Response and Analysis 


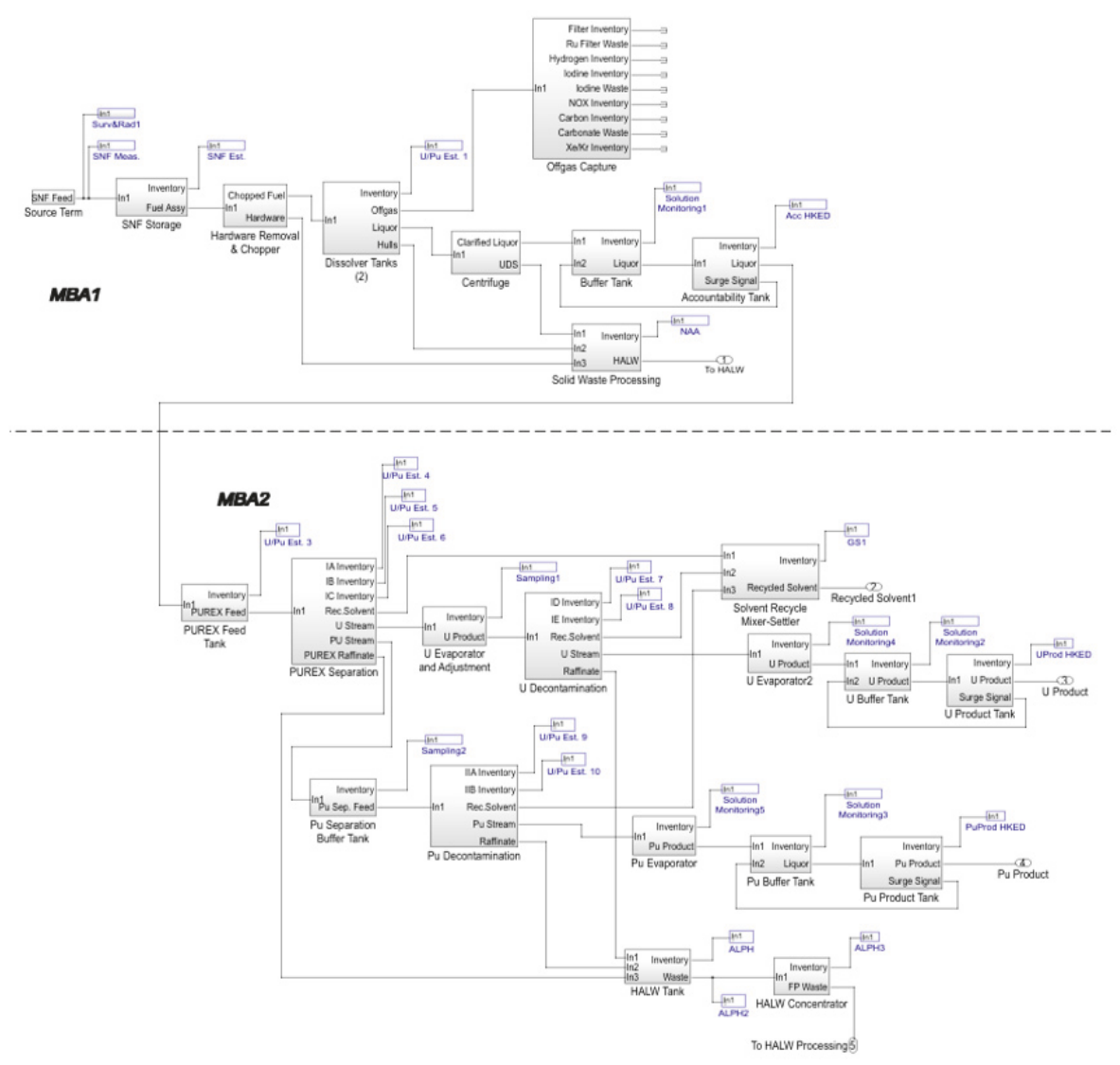

FIGURE 1: SSPM for a PUREX reprocessing plant.

Software) for simulation of gamma spectra. The blue blocks represent measurement points and feed into an overall material balance calculation. Diversion blocks are used to simulate various types of material loss scenarios. The operation of the model and data output is controlled with a graphical user interface.

An electrochemical SSPM version is shown in Figure 2. The operation of the model is similar, but with differences appropriate to molten salt processing of nuclear material. All models contain the ability to turn on material diversions from various locations to examine the effect on overall plant safeguards. While the models include a significant amount of detail on the plant operations, the main purpose is for the simulation of the safeguards measurements. Much of the past work [2] has focused on the development of an appropriate material balance calculation and statistical tests to determine probabilities of detection.

\section{Traditional Safeguards Approach}

The traditional safeguards approach requires taking precision measurements of inputs, outputs, and the change in inventories in each MBA. The SSPM simulates the various measurement technologies by choosing what is measured, the measurement uncertainties, and the timing. For example, an input accountability tank may use both a sample, which is measured using Hybrid K-Edge Densitometry, and a level measurement. The model will simulate both measurements to determine an overall actinide measurement, with appropriate error propagation.

All the measurements in the model are used to set up an inventory difference, or material unaccounted for (MUF) calculation. This usually involves one input measurement, several inventory measurements, and multiple output measurements. The SSPM tracks all this data over a given material balance period. Timing is an important consideration because measurements are taken at different times. Delay blocks are used to synchronize the measurements for a particular point in time. The input and output accountability batches are summed over the material balance period, and the difference of the inventories from the previous measurement point are used. Again, error propagation is taken into account.

This data is all fed into an embedded function block that uses MATLAB script to calculate MUF and $\sigma_{\text {MUF }}$, the overall error. The main statistical test that is used in a joint Page's test; this includes both an abrupt and protracted test to increase the probability of detecting both types of losses. The actual test is a Page's test of SITMUF (Standardized Independent Transformed MUF) [5]. Past work, both with this model and by other researchers, has shown the robustness of this test for detecting a variety of material loss patterns [6-8]. 


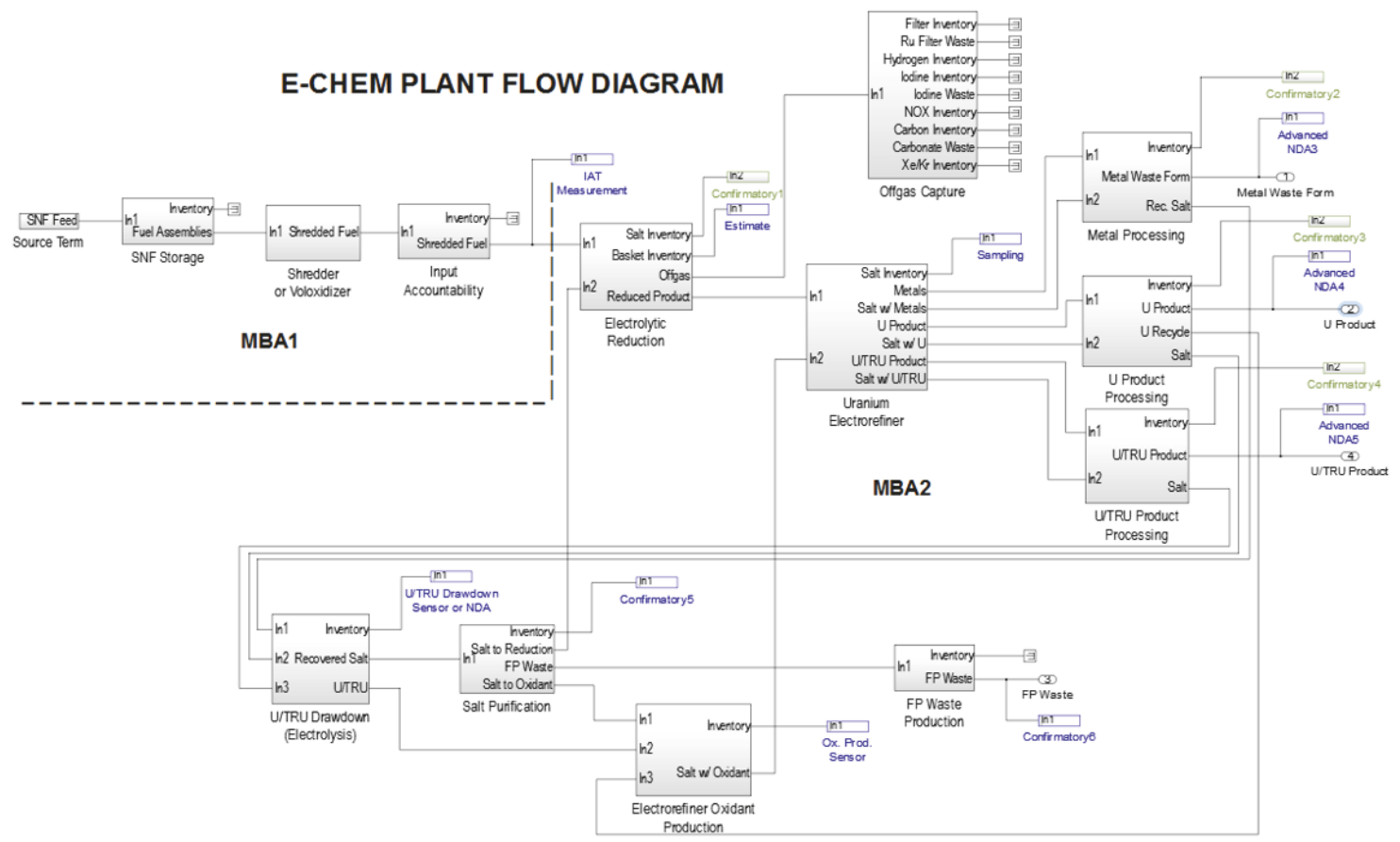

FIGURE 2: SSPM for an electrochemical reprocessing plant.

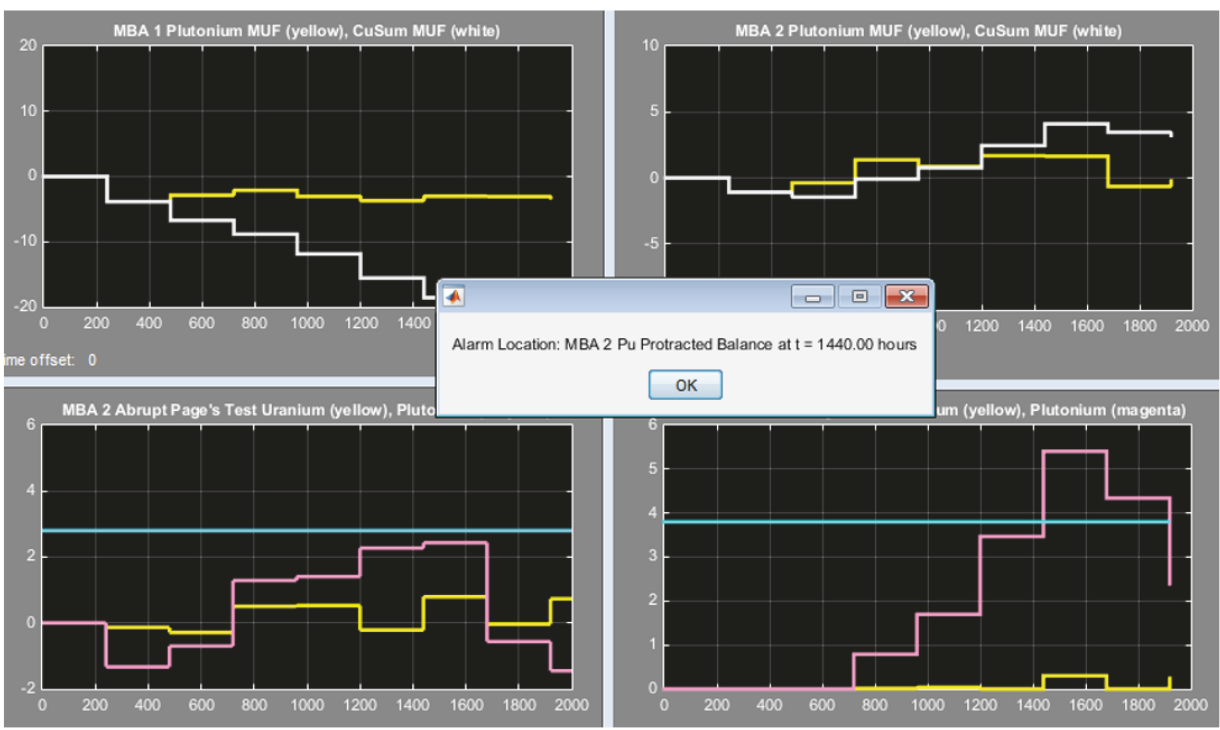

FIGURE 3: Example protracted loss scenario using the SSPM. The top plots show the MUF and cumulative sum of MUF for Pu for two MBAs, and the bottom plots show the abrupt and protracted Page's test. The Page's test threshold (lower right) was surpassed, indicating a diversion.

Additional statistical tests are also included in the SSPM. The Shewhart, CuMUF (Cumulative Sum of MUF), and GeMUF (geschätzter, or estimated, MUF) [9] tests are also included. The Shewhart test was first proposed by Walter Shewhart in the 1920s and is useful for detecting abrupt loss. The CuMUF test performs the best for detection loss spread out over several balance areas. The GeMUF test is comparable to Page's test in having a high detection probability for a variety of diversion scenarios.
3.1. Example Modeling Results. The model can evaluate diversion scenarios as a stand-alone run or through the cumulation of results of multiple iterations. Figure 3 shows an example of protracted material loss for a PUREX facility. The top plots show the MUF and cumulative sum of the MUF for MBA1 and MBA2. It is difficult to see a diversion in this data. The lower plots show the results of the abrupt and protracted Page's test for the same run; the protracted test indicated an alarm when the pink line surpassed the blue line threshold. 


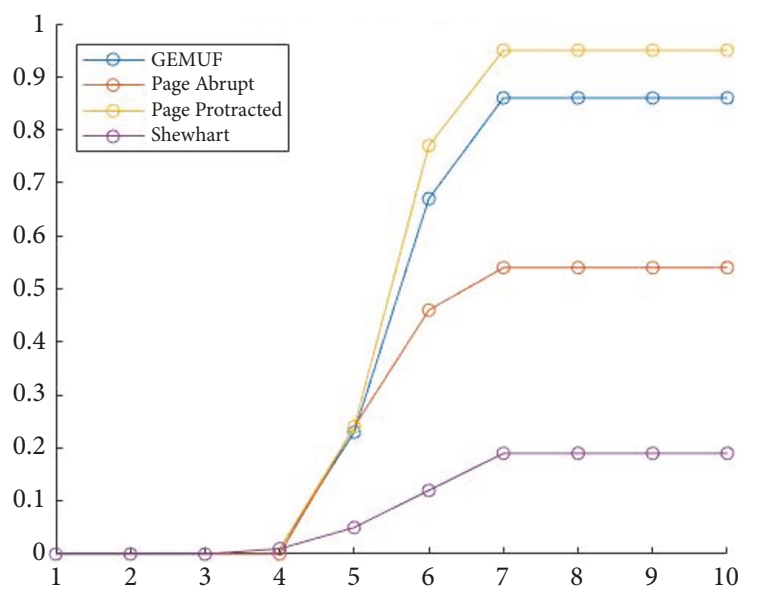

FIGURE 4: Comparison of statistical tests for a protracted material loss. The $\mathrm{y}$-axis represents the detection probability, and the $\mathrm{x}$-axis is the material balance period.

Every model run is slightly different due to the random nature of the way errors are applied to the measurements. Multiple iterations are run to develop detection probability statistics.

The SSPM has also been used to compare statistical tests. Figure 4 shows an example of a comparison for a protracted loss. The Page's protracted test performed the best, followed by GeMUF, with much lower detection probability for the Page's abrupt and Shewhart tests.

The SSPM has been used for various analyses using a traditional safeguards approach like shown here. These analyses include evaluating the effect of better measurements, changing the material balance period, and testing different statistical tests. The SSPM provides a very visual and rapid systems level analysis that would be too difficult and expensive to evaluate experimentally. The next section discusses alternative safeguards approaches which require using simulation data in different ways.

\section{Alternative Safeguards Approach}

Reliance on precision measurements for traditional materials accountancy usually requires sampling and destructive analysis. Laboratory analysis is expensive and places financial burden on both the operator and inspector. For example, international safeguards for the Rokkasho Reprocessing Plant in Japan required the International Atomic Energy Agency (IAEA) to build an on-site laboratory. It is probably not feasible for IAEA to have several on-site laboratories if additional future facilities are built. There is a strong desire to move away from laboratory analysis and instead rely more on unattended measurements to reduce the burden of safeguards.

The difficulty with this approach is that on-line or unattended measurements typically have higher measurement uncertainties. For large bulk handling facilities, measurement uncertainties much higher than 1-2\% for the actinides will mean that the facility will not be able to meet IAEA accountancy goals. Despite that, newer on-line measurements and process monitoring data show promise in being able to detect changes in plant conditions, even for very protracted diversion scenarios. These measurements require a different safeguards approach that relies on a machine learning algorithm, which can only be developed through simulation. The following sections describe the alternative approach.

4.1. Examination of Types of Diversion Scenarios. The alternative approach focuses less on an overall actinide balance and more on addressing IAEA goals: timely detection of diversion or undeclared production or processing of nuclear material. There are two ways material can be removed: through direct and substitution loss. Direct loss means that material is directly removed from the process; bulk mass measurements can detect this loss well. Precision scales and electromanometers can determine mass with $0.1 \%$ measurement uncertainty, so direct material loss (whether abrupt or protracted) can often be detected rapidly. Substitution loss requires removing material and replacing it with a surrogate designed to defeat the bulk balance.

It is relatively straight-forward to set up a bulk balance to detect direct loss. The Solution Measurement and Monitoring System (SMMS) at Rokkasho [10] is a system comprised of both joint-use and operator-owned electromanometers to keep track of bulk material in the main separation area of the plant. The measurements are unattended, so ideal for IAEA use.

Since direct loss can easily be detected, the challenge becomes detecting substitution losses without high-precision actinide measurements. This is where modeling and simulation plays a strong role. Using the SSPM, these substitution diversion scenarios can be set up to examine changes in plant conditions, and specifically how or if NDA or on-line measurements can be used to detect the loss. An actinide balance by itself does not need to detect the loss as long as there is some way to detect that a substitution diversion has occurred. For example, the removal of molten salt in a pyroprocessing plant, followed by replacement with clean salt will lead to a change in a fission product gamma spectra. A gamma measurement may only be able to achieve 5$10 \%$ uncertainty for quantifying $\mathrm{Pu}$, so small losses can slip through. However, a gamma measurement may be able to detect the drop off of a Cs peak to $1 \%$ measurement uncertainty which could indicate a salt removal.

4.2. Testing the Approach Through Modeling. The alternative safeguards approach was examined using the SSPM for a generic pyroprocessing facility. Since bulk balances typically can easily detect direct material loss, the focus of the analysis was on substitution diversions. This work utilized the integration of the GADRAS code [4] with the SSPM for generating gamma spectra based on the isotopic inventories.

The use of gamma measurements have challenges associated with them. Cs usually dominates any gamma spectra, so the applicability will vary by location. Self-shielding is a problem for large products, so a gamma measurement likely will need to be done using uniform samples. There is also variability in the isotopic content since reprocessing plants process a mixture of fuel types. All of these issues make it 


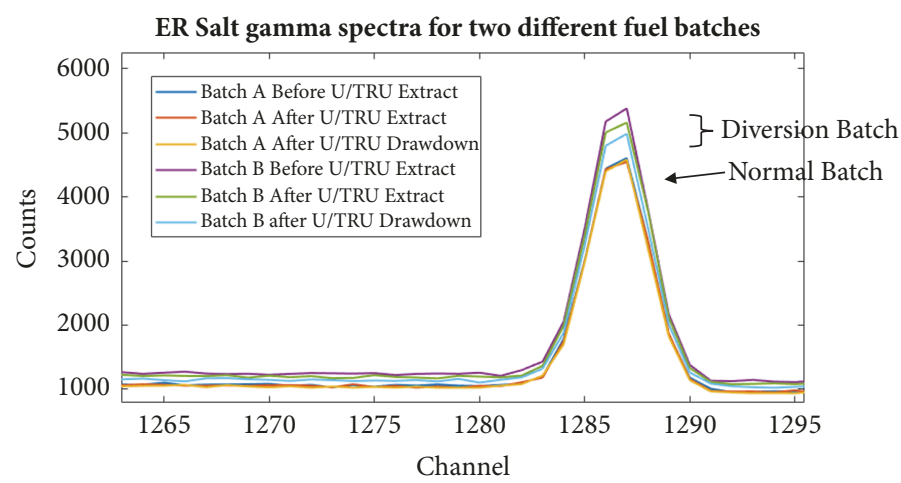

FIGURE 5: ER salt gamma peak during one normal batch and one substitution diversion.

incredibly important to have a model with sufficient detail and variability that would be representative of an actual facility.

One example will be shown here to demonstrate the concept. The SSPM was used to model a substitution diversion of electrorefiner salt as compared to a normal batch. A gamma analysis of the salt before, during, and after the main set of extractions was used to compare the diversion case with the normal case. Figure 5 shows one particular gamma peak for the normal and diversion batches. Due to the variability in the fuel feed, it is expected that there will be variation in the peak size between batches. The indication of diversion is shown as a change of the peak height within one particular batch.

Other substitution diversions are being examined as well. The difficulty with this approach is that it can require looking at several different possible substitution diversions and analyzing each one for potential indicators. It is possible that several gamma peaks, or ratios of peaks in different areas, or entirely different measurements will need to be analyzed. The SSPM makes this possible, but these indicators then need to be built into a machine learning algorithm.

4.3. Machine Learning. The One Class Support Vector Machine (OCSVM) [11] is an unsupervised machine learning technique that has promise for this application. It can be trained with normal data only, which is desirable since actual fuel cycle facilities will not be able to provide off-normal data for diversion scenarios. The OCSVM can take any number of input data streams and generate a boundary around normal data such that some defined percentage of points fits inside the boundary. Using normal data, the boundary can be set to achieve a desired false alarm probability. The OCSVM will produce a classification of 1 (normal) or -1 (off-normal) at each point in time that the calculation is applied. An alarm may be reached when a certain number of off-normal classifications occur in a row.

The SSPM is being used to generate large data sets of normal data to train the machine learning algorithm. Then the SSPM will be used to test the algorithm using various direct and substitution diversion scenarios. The results will be compared to detection using more traditional approaches.

Figure 6 illustrates how the OCSVM technique works. Several bulk mass measurements and NDA or process

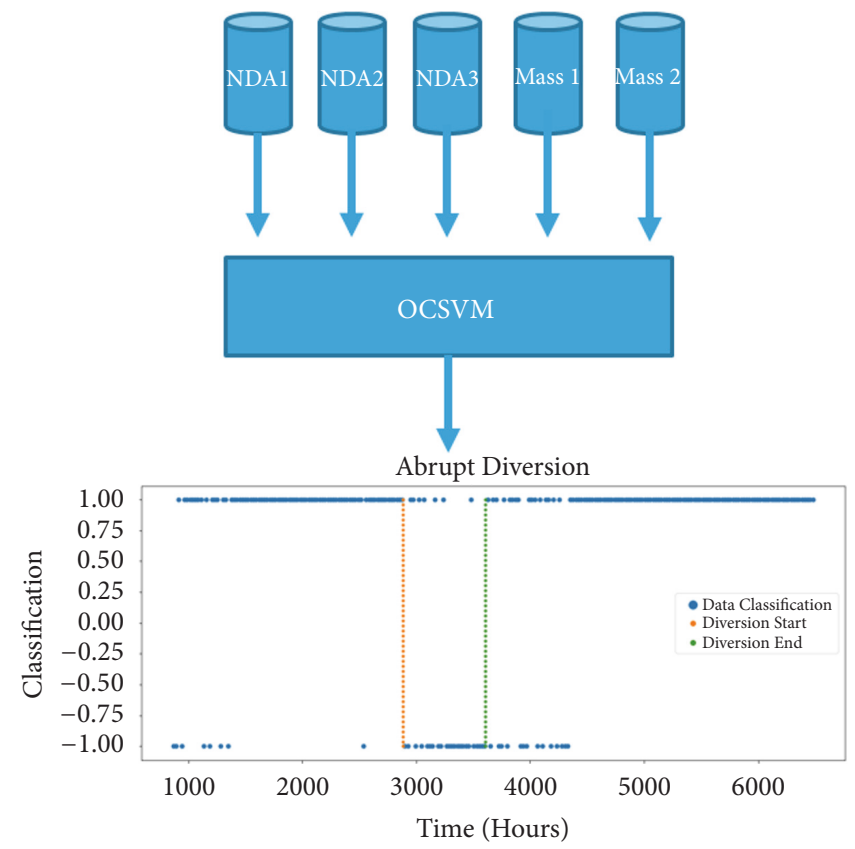

FIGURE 6: Illustration of the OCSVM technique.

monitoring measurements will be fed into it. During normal operation, the OCSVM will generate mostly +1 classifications, but some misclassifications (-1) are expected. During an abrupt diversion, the frequency of misclassifications significantly increases. An appropriate threshold can be set on the frequency of misclassifications in a set time period.

Significantly more work is required to test this approach. The previous discussion illustrates how much simulation data is required. Future work will show results as compared to a traditional material balance.

\section{Conclusion}

The SSPM is a versatile facility safeguards modeling tool that is being used to analyze a variety of safeguards challenges. It has been used for analysis of safeguards metrics using traditional material balances to help inform measurement 
technology requirements. It has also been used for diversion scenario analysis to prove safeguards design.

The alternative safeguards approach presented here attempts to develop a way to safeguard a facility with reliance only on unattended measurements. This concept is yet to be proven but could be of significant benefit to IAEA. The alternative approach requires a large amount of modeling data to determine the indicators, train the machine learning algorithm, and test the algorithm under diversion scenarios. The SSPM provides enough fidelity to be used for this purpose.

The alternative approach is more feasible for a new facility, where the technology can be built into the plant. It would be difficult to apply these changes to an existing, operating facility. The approach requires a different way of thinking about the problem by breaking it down into the detection of direct and substitution diversion scenarios. The preliminary results show promise in being able to detect indications of small substitution diversions using NDA measurements. A much deeper dive of the concept and training of a machine learning algorithm will be required to move this work forward.

\section{Data Availability}

The data used for the preliminary results in this work can be accessed by contacting the author.

\section{Disclosure}

Sandia National Laboratories is a multimission laboratory managed and operated by National Technology and Engineering Solutions of Sandia, LLC, a wholly owned subsidiary of Honeywell International, Inc., for the US Department of Energy's National Nuclear Security Administration under Contract DE-NA-0003525. This paper describes objective technical results and analysis. Any subjective views or opinions that might be expressed in the paper do not necessarily represent the views of the US Department of Energy or the United States Government.

\section{Conflicts of Interest}

The authors declare that there are no conflicts of interest regarding the publication of this paper.

\section{Acknowledgments}

The modeling development was funded through the Fuel Cycle Technologies program under the US Department of Energy Office of Nuclear Energy.

\section{References}

[1] B. B. Cipiti, F. A. Duran, B. R. Key, Y. Liu, I. Lozano, and R. Ward, "Modeling and design of integrated safeguards and security for an electrochemical reprocessing facility.” Tech. Rep. SAND2012-9303, 2012.
[2] B. B. Cipiti, "Separations and safeguards performance modeling for advanced reprocessing facility design," Journal of Nuclear Materials Management, vol. 39, no. 2, pp. 4-14, 2011.

[3] W. Wieselquist, "ORIGEN: Neutron activation, actinide transmutation, fission product generation, and radiation source term calculation," SCALE Manual, pp. 3-5, 2016.

[4] S. M. Horne, GADRAS-DRF 18.6 Users Manual, SAND20164345, Sandia National Laboratories, 2016.

[5] B. B. Cipiti, M. McDaniel, and T. Burr, "Incorporation of the Pages Test in the Separation and Safeguards Performance Model," INMM Information Analysis Workshop, 2014.

[6] T. P. Speed and D. Culpin, "The Role of Statistics in Nuclear Materials Accounting: Issues and Problems," Journal of the Royal Statistical Society A, 149/4 pp. 281-313, 1986.

[7] B. J. Jones, "Near Real Time Materials Accountancy using SITMUF and a Joint Pages Test: Comparison with MUF and CUMUF Tests," ESARDA Bulletin, vol. 15, 1988.

[8] T. Burr, M. S. Hamada, M. Skurikhin, and B. Weaver, "Pattern Recognition Options to Combine Process Monitoring and Material Accounting Data in Nuclear Safeguards," Statistics Research Letters, vol. 1, no. 1, pp. 6-31, 2012.

[9] R. Seifert, "The GEMUF Test: A New Sequential Test for Detecting Loss of Material In a Sequence of Accounting Periods," IAEA-SM-293/46, 1986.

[10] S. J. Johnson and M. Ehinger, "Designing and Operating for Safeguards: Lessons Learned From the Rokkasho Reprocessing Plant (RRP)," Tech. Rep. PNNL-19626, 2010.

[11] B. Scholkopf, "Estimating the Support of High-Dimensional Distribution," Microsoft Research, pp. 99-87, 1999. 

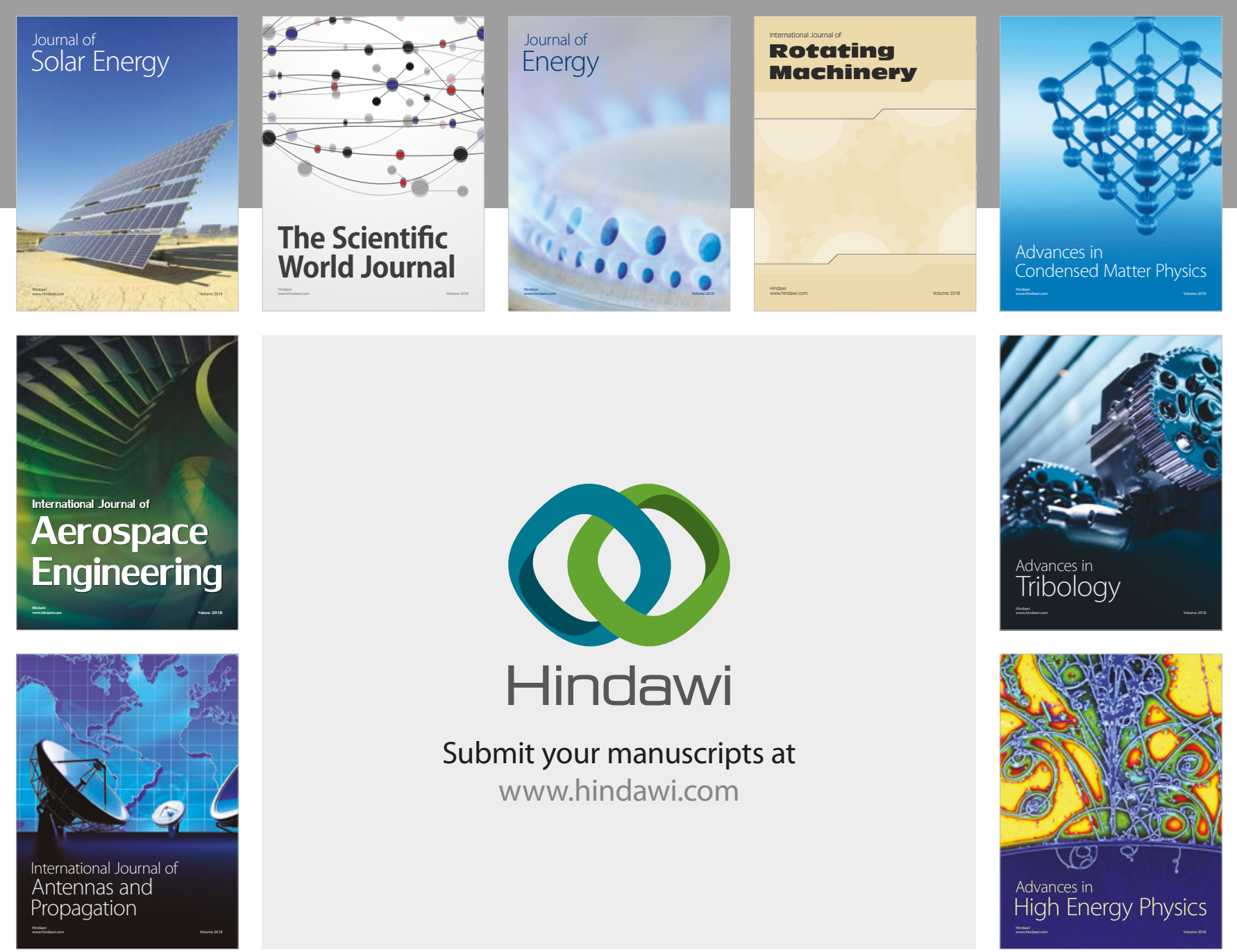

Submit your manuscripts at

www.hindawi.com
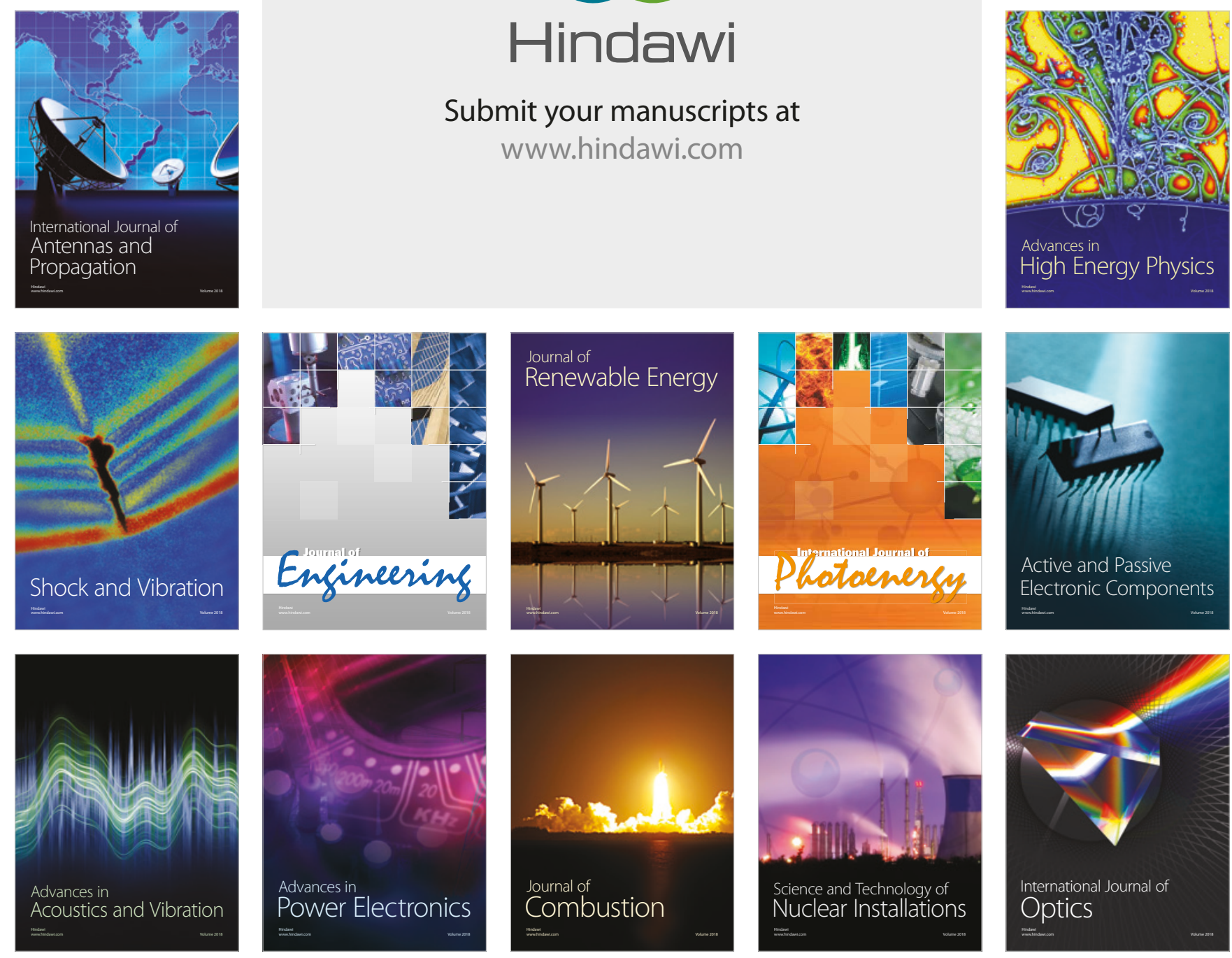